\title{
Correction to: Minimally Invasive Aesthetic Procedures
}

\author{
Adilson Da Costa
}

\section{Correction to:}

\section{A. Da Costa (ed.), Minimally Invasive Aesthetic Procedures, https://doi.org/10.1007/978-3-319-78265-2}

The "Tip Box" content for Chapters 93 and 96 was inadvertently published incorrectly. This has been corrected in the current version of the book. 